Abanico Veterinario. Enero-Diciembre 2021; 11:1-16. http://dx.doi.org/10.21929/abavet2021.20

Artículo Original. Recibido: 08/12/2020. Aceptado: 31/03/2021. Publicado: 20/04/2021. Clave: e2020-100.

\title{
Influencias climáticas, regionales y cantidad de cría en el comportamiento higiénico de Apis mellifera
}

\author{
Climatic, regional, and bee brood quantity influences on the hygienic behavior of Apis \\ mellifera.
}

\section{Tapia-González José ${ }^{\star 1,4}$ ID, León-Mantecón Talit ${ }^{1}$, Contreras-Escareño Francisca $^{2,4}$ ID , Macias-Macias José1,4 ID, Tapia-Rivera José ${ }^{\star * 1,4 ~ I D, ~ G u z m a ́ n-N o v o a ~}$ Ernesto ${ }^{1,3,4}$ ID}

1Departamento de Ciencias Económicas y Administrativas. Centro Universitario del Sur. Universidad de Guadalajara. México, C.P. 49000. ${ }^{2}$ Departamento de Producción Agrícola, Centro Universitario de la Costa Sur. Universidad de Guadalajara. México, C.P. 48900. ${ }^{3}$ School of Environmental Sciences, University of Guelph, 50 Stone Road East, Guelph, N1G 2W1, Ontario, Canada. ${ }^{4}$ Centro en Investigaciones en Abejas, Centro Universitario del Sur. Universidad de Guadalajara. México, C.P. 49000. *Autor responsable: José María Tapia González, ${ }^{*}$ Autor de correspondencia: José Carlos Tapia Rivera, Dirección postal: Centro Universitario del Sur Av. Enrique Arreola Silva No. 883, colonia centro C.P. 49000, Ciudad Guzmán, Jalisco, México. Tel: 3415752222 ext: 46087. joset@cusur.udg.mx, talit89@hotmail.com, francisca.contreras@academicos.udg.mx, joseoc@cusur.udg.mx, jose.tapia@cusur.udg.mx, eguzman@uoguelph.ca

\section{RESUMEN}

El comportamiento higiénico de las abejas (Apis mellífera) es un mecanismo altamente influenciado por efectos genéticos que les confiere resistencia contra enfermedades y parásitos. El objetivo de este estudio fue determinar si las influencias climáticas, regionales y de cantidad de cría se relacionan con el comportamiento higiénico en colmenas del estado de Jalisco, México. Se evaluaron 142 colonias mediante el método de congelación de pupas con nitrógeno líquido en dos zonas climáticas (zonas montaña y valle). Las colonias ubicadas en los municipios de la zona baja removieron significativamente más cría congelada que las colonias ubicadas en los municipios de la zona alta $(p<0.001)$ y estas diferencias estuvieron significativamente correlacionadas con la temperatura ambiental $(r=0.25, p<0.01)$ y la altura sobre el nivel del mar (asnm) $(r=-0.27, p<0.001)$. También se encontró que en la región más cálida y de menor altitud, un tercio de las colonias fueron altamente higiénicas (> 80\%). Este grado de limpieza es aceptable para iniciar proyectos de crianza selectiva de abejas altamente higiénicas para mejorar la sanidad de las colonias, lo cual beneficiaría a la industria apícola.

Palabras Clave: Apis mellífera, comportamiento higiénico, reproducción, clima, altitud.

\begin{abstract}
The hygienic behavior of honeybees (Apis mellífera) is a mechanism highly influenced by genetic effects that confers resistance against diseases and parasites. The objective of this study was to determine whether climatic, regional and brood quantity influences are related to hygienic behavior in beehives in the state of Jalisco, Mexico. A total of 142 colonies were evaluated by freezing pupae with liquid nitrogen in two climatic zones (mountain and valley). Colonies located in the municipalities of the low zone removed significantly more frozen brood than the colonies located in the municipalities of the higher zone $(p<0.001)$, and these differences were significantly correlated with environmental temperature $(r=0.25, p<0.01)$ and altitude above sea level $(r=-0.27, p<0.001)$. It was also found that in the warmer, lower altitude region, one third of the colonies were highly hygienic $(>80 \%)$. This degree of cleanliness is acceptable for initiating selective breeding projects for highly hygienic bees to improve colony health, which would benefit the beekeeping industry.
\end{abstract}

Keywords: Apis mellifera, hygienic behavior, reproduction, climate, altitude. 


\section{INTRODUCCIÓN}

México es uno de los principales países productores y exportadores de miel de abejas (Apis mellifera) en el mundo y la apicultura es una actividad de enorme importancia socioeconómica para el país. A ella se dedican cerca de 45,000 productores, quienes trabajan alrededor de 1.9 millones de colonias (Magaña et al., 2016). Sin embargo, en años recientes la producción de miel no se ha incrementado por falta de procesos que puedan potencializar este sector (Contreras-Escareño et al., 2013; Soto-Muciño et al., 2017). La apicultura mexicana es afectada por diversos factores que reducen la productividad y rentabilidad de las colonias, entre los que se incluyen enfermedades de las abejas ocasionadas por diversos microorganismos y parásitos (Magaña-Magaña et al., 2016). En Jalisco, uno de los principales estados productores de miel de México, prevalecen enfermedades que afectan a la cría y abejas adultas, como la nosemosis, varroosis y ascosferosis (Tapia-González et al., 2017, 2019, 2020). Estos problemas sanitarios pueden producir pérdidas económicas de muchos millones de pesos anualmente, pero las pérdidas pudieran reducirse si los apicultores establecieran programas de crianza selectiva para desarrollar estirpes de abejas resistentes a enfermedades y parásitos.

Uno de los mecanismos de resistencia a enfermedades y parásitos de las abejas melíferas más conocido es el comportamiento higiénico, el cual consiste en la capacidad de las abejas de detectar larvas y pupas muertas, enfermas, o parasitadas con ácaros de Varroa destructor, con el fin de abrir las celdas para limpiarlas. Por lo tanto mediante este mecanismo esencial se reducen los riesgos de transmisión de patógenos y parásitos (Emsen et al., 2012). El comportamiento higiénico es importante en insectos sociales por el hacinamiento en que viven, ya que, si un individuo se enferma, la posibilidad de transmisión entre integrantes de la colonia es muy alta (Guzman-Novoa y Morfin, 2019). Además, las colonias de abejas con alto comportamiento higiénico pueden incrementar la producción de miel (Wielewski et al., 2012).

El comportamiento higiénico de las abejas melíferas es afectado por efectos genéticos y es heredable (Arechavaleta-Velasco et al., 2011; Pernal et al., 2012), por lo que es viable desarrollar estirpes de abejas altamente higiénicas con programas de mejoramiento genético (Ivernizzi et al., 2011). La expresión del comportamiento higiénico es variable (Xonis et al., 2015), lo que se debe en parte a los efectos genéticos antes mencionados, pero también a la influencia de factores ambientales. Poco se sabe sobre los efectos ambientales que afectan el comportamiento higiénico de las abejas melíferas, pero se ha reportado que la cantidad de cría de la colonia y cambios en el flujo de néctar recolectado por las abejas pueden afectarlo (Wagoner et al., 2018), otros estudios apoyan la idea del comportamiento de las abejas (Sousa et al., 2016). Es importante determinar qué factores ambientales influyen en el comportamiento higiénico de las abejas melíferas para estandarizar sus efectos cuando se establezcan programas de crianza selectiva para 
desarrollar estirpes de abejas altamente higiénicas. En este sentido el objetivo de este estudio fue determinar si las influencias climáticas, regionales y de cantidad de cría se relacionan con el comportamiento higiénico en colmenas del estado de Jalisco, México.

\section{MATERIAL Y MÉTODOS}

Regiones de estudio. El presente trabajo se realizó en apiarios de siete municipios del estado de Jalisco, México, ubicados en una región alta o de montaña (Tapalpa y Unión de Guadalupe) y en una región baja o de llano (Tamazula de Gordiano, Zapotlán el Grande, Sayula, San Martín Hidalgo y Zacoalco de Torres). El clima y altitud sobre el nivel del mar (asnm) presentan grandes contrastes debido a la conformación variada de relieves de estas regiones (Plan Regional de Desarrollo, 2012). Se utilizaron 142 colonias de 45 apiarios para evaluar su comportamiento higiénico y capacidad reproductiva en los siete municipios mencionados (Tabla1) durante la época de estiaje (entre marzo y mayo).

Tabla 1. Número de colonias y apiarios de abejas melíferas evaluadas para comportamiento higiénico por municipio, en regiones de montaña y llano, de Jalisco, México

\begin{tabular}{cccc}
\hline Municipio & Región & No. colonias & No. apiarios \\
\hline Tapalpa & Montaña & 27 & 9 \\
\hline Unión de Guadalupe & Montaña & 28 & 9 \\
Tamazula & Llano & 17 & 5 \\
\hline Zapotlán el Grande & Llano & 14 & 7 \\
\hline Sayula & Llano & 22 & 6 \\
\hline San Martin Hidalgo & Llano & 18 & 5 \\
\hline Zacoalco & Llano & 16 & 45
\end{tabular}

Unidades experimentales. De cada apiario de los siete municipios se seleccionaron al azar y se marcaron de tres a cuatro colonias, las cuales fueron identificadas con un disco de plástico donde se registró el número de la colonia y se llevaron registros de localización. Cada colonia seleccionada se abrió y se inspeccionó para asegurarse que visualmente no se detectaran signos de enfermedades de la cría (loque americana, loque europea, cría calcárea, o cría ensacada). Las colonias que presentaron algún indicio de enfermedad no fueron usadas. 
Evaluación del comportamiento higiénico. De cada una de las colonias seleccionadas se eligió el bastidor de cría con la mayor cantidad de celdas operculadas con patrón continuo y uniforme. El bastidor elegido se colocó en posición horizontal arriba de la colmena, y sobre él se puso un cilindro sin tapa de policloruro de vinilo (PVC) de $10 \times 11$ $\mathrm{cm}$, ejerciendo ligera presión con movimientos en círculo sobre la cría operculada. Se calculó que este cilindro cubrió una cantidad promedio de 350 celdas. Posteriormente se procedió a sacrificar la cría dentro del cilindro por medio de congelamiento (Spivak, 1996). Para ello se vaciaron dentro de este cilindro $250 \mathrm{ml}$ de nitrógeno líquido $\left(-195^{\circ} \mathrm{C}\right)$ usando un vertedor de aluminio. Una vez terminado el proceso de evaporación del nitrógeno (aprox. $1 \mathrm{~min}$ ), se tomó una imagen digital (cámara digital Nikkon, Coolpix) del área de cría congelada para posteriormente contar el número de celdas iniciales. El bastidor experimental fue identificado con el número de la colonia, marcándolo con plumón indeleble sobre el cabezal. Posteriormente, el bastidor se introdujo al centro de la cámara de cría de la colonia de origen para que las obreras iniciaran la remoción de la cría sacrificada (comportamiento higiénico). Después de $24 \mathrm{~h}$ de este procedimiento, se volvió a abrir la colmena y se extrajo el bastidor con la cría que se congeló. Se tomó una segunda fotografía del área congelada para contar el número de celdas limpias (donde las abejas retiraron pupas). Luego, usando los números de celdas de ambas fotografías, se determinó el porcentaje de cría removida (Espinosa-Montaño et al., 2008), este procedimiento se realizó en una ocasión. Se utilizó la siguiente fórmula para obtener el porcentaje de remoción de cría:

$$
\text { Porcentaje de remoción de cría }=\frac{\text { Número de pupas removidas }}{\text { Total de celdas congeladas }}(100)
$$

Determinación de la capacidad reproductiva de las colonias. Para determinar la capacidad reproductiva, en cada colonia se calculó el número de bastidores con cría operculada mediante la estimación de la proporción del área de cada panal que estaba cubierto con cría sellada (0-1.0) y se sumaron estos valores (Delaplane et al., 2013).

Determinación de altitud y variables climáticas. Para medir la altitud sobre el nivel del mar (asnm) de cada apiario, se usó un GPS (Sportrack-color, Magellan). Los datos de temperatura y humedad ambiental promedio se obtuvieron de la página electrónica, municipios de Jalisco (Gobierno del estado de Jalisco, 2019).

Análisis estadísticos. Los datos de porcentaje de comportamiento higiénico se transformaron al arcoseno de la raíz cuadrada para normalizarlos como lo sugiere William (1990) y Medina-Flores et al (2019). Los datos de número de panales con cría requirieron transformación logarítmica y los datos climáticos no fueron transformados por presentar una distribución normal, para definir la normalidad de los datos se utilizó la prueba de 
Kolmogorov-Smirnov (Hanusz y Tarasińska, 2015). Las respuestas higiénicas de las colonias, así como la cantidad de cría y los parámetros climáticos se analizaron entre municipios por medio de análisis de varianza. Al encontrarse significancia, las medias fueron separadas y comparadas con pruebas protegidas de Fisher $(p<0.05)$. Los datos entre regiones se compararon con pruebas $t$ de student. También, con pruebas de Pearson, se correlacionó la respuesta higiénica de las colonias con los datos de cantidad de cría y de los parámetros climáticos. Además, se crearon histogramas de distribución de frecuencia con el fin de determinar el porcentaje de colonias con bajo y alto comportamiento higiénico para las colonias de cada región. Los análisis se realizaron con el paquete estadístico SPSS versión $24 \AA$ (Quezada-Lucio, 2017).

\section{RESULTADOS}

El grado de comportamiento higiénico de las colonias de abejas varió significativamente entre municipios $\left(F_{6,135}=4.88, p<0.001\right)$ y entre regiones $\left(t_{140}=-4.05, p<0.001\right)$. Las colonias ubicadas en los municipios de la región del llano fueron significativamente más higiénicas que las colonias ubicadas en los municipios de montaña (Figuras 1, 2).

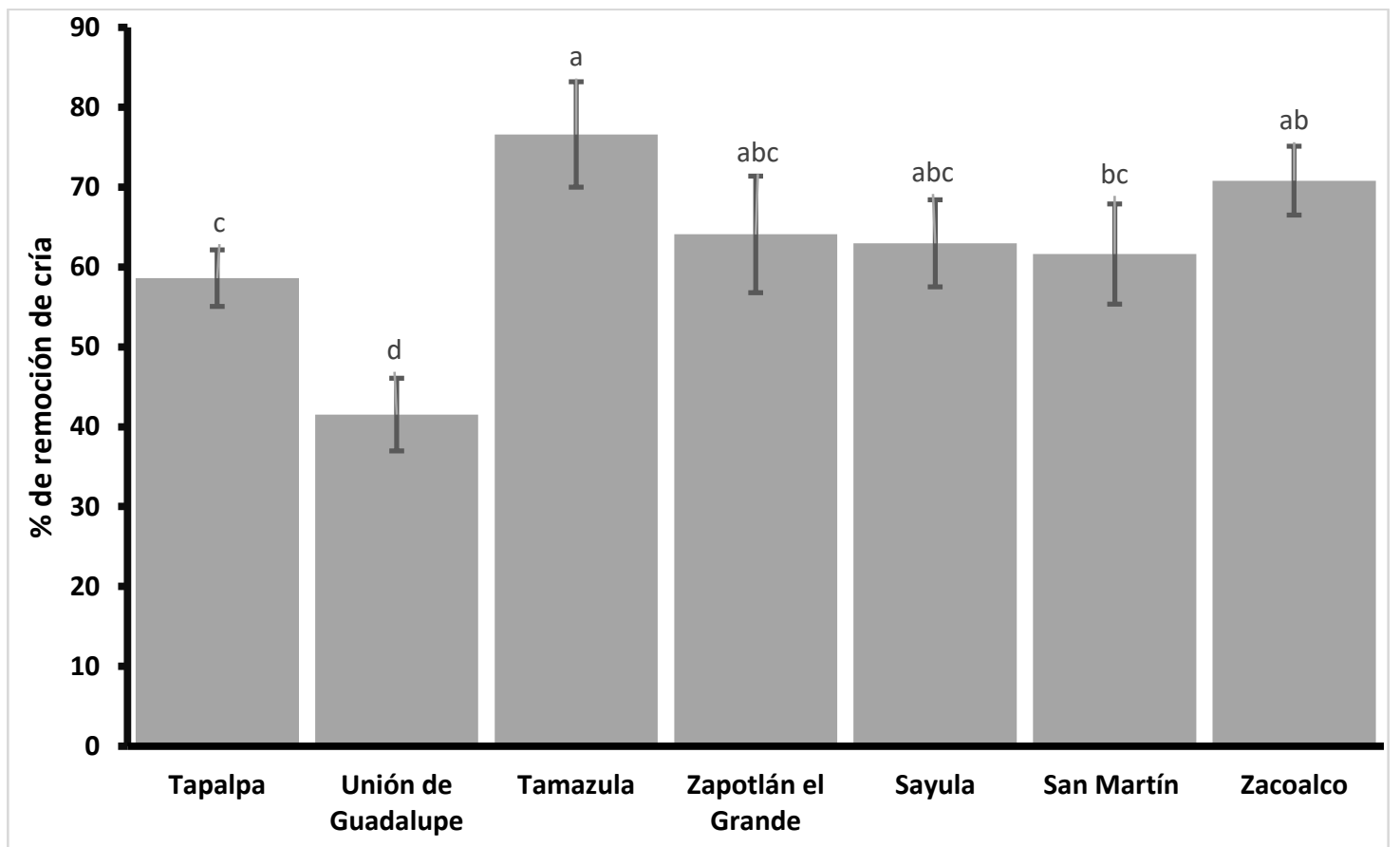

Letras diferentes sobre las barras significan diferencias significativas en base a análisis de varianza y pruebas de Fisher.

Figura 1. Porcentaje de remoción de cría \pm E.E. de 142 colonias de abejas en siete municipios 


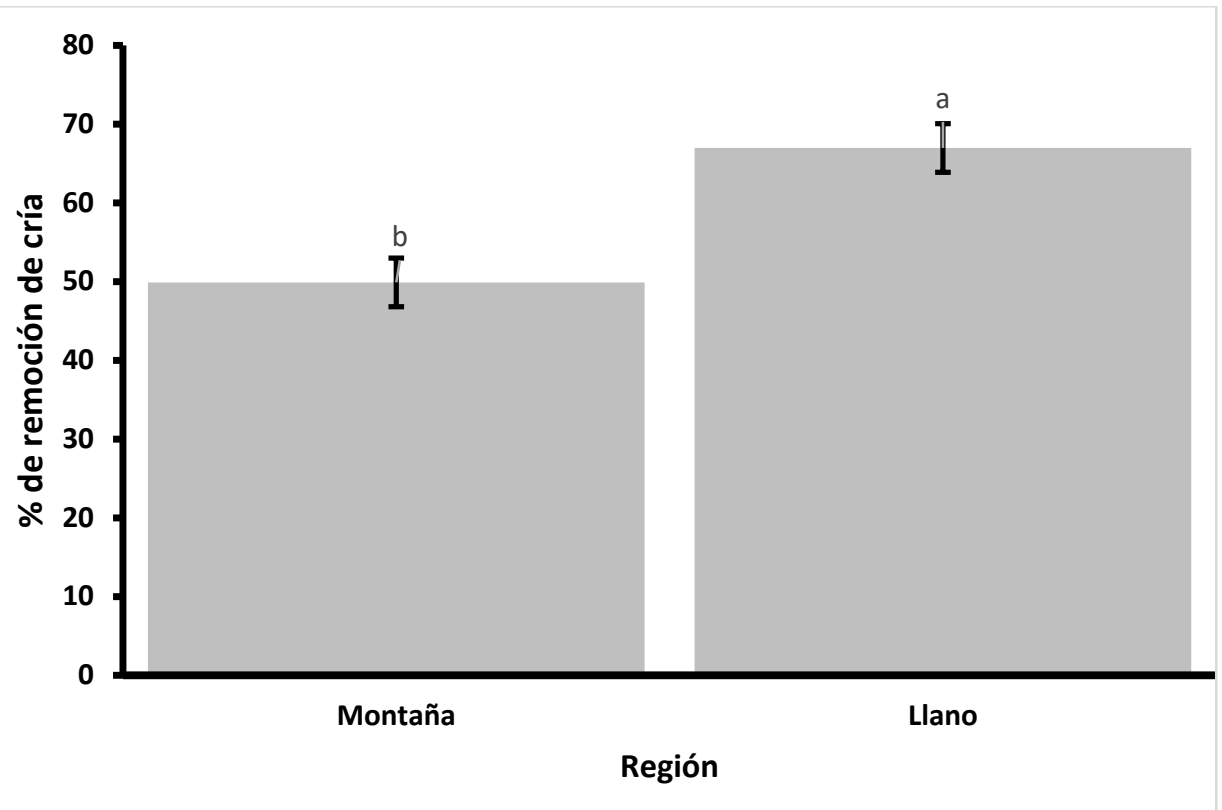

Figura 2. Porcentaje de remoción de cría \pm E.E. de 142 colonias de abejas en dos regiones. Letras diferentes sobre las barras significan diferencias significativas en base a una prueba t de student

Se encontraron diferencias significativas en la cantidad de cría de las colonias, así como en la temperatura y humedad relativa entre municipios $\left(F_{6,135}=4.94, F_{6,135}=70.28, F_{6,135}\right.$ $=63.07, p<0.0001$, respectivamente). También se observaron diferencias significativas para la temperatura entre regiones $\left(\mathrm{t}_{140}=-10.50, \mathrm{p}<0.0001\right)$, pero no para humedad relativa y cantidad de cría ( $\mathrm{t}_{140}=-0.31$ y $\mathrm{t}_{140}=-0.32, \mathrm{p}>0.75$, respectivamente). Para los factores de cantidad de cría y humedad relativa, no se observó un patrón relacionado con la región o con la asnm. Hubo variación similar en los municipios de ambas regiones para estos dos factores. Para el factor temperatura, las colonias ubicadas en los municipios de la zona del llano tuvieron un clima significativamente más cálido que las colonias ubicadas en los municipios de montaña (Tablas 2, 3).

Tabla 2. Medias \pm E.E. de cantidad de cría (número de panales) y de factores climáticos y ambientales (altura sobre el nivel del mar: asnm) de las colonias de abejas evaluadas para comportamiento higiénico por municipio

\begin{tabular}{lllll}
\hline Municipio & Panales c/cría & Temperatura & Humedad Rel. & asnm \\
\hline Tapalpa & $4.9 \pm 0.2^{\mathrm{c}}$ & $29.2 \pm 0.3^{\mathrm{c}}$ & $24.2 \pm 0.6^{\mathrm{c}}$ & $2119 \pm 22$ \\
Unión de Guadalupe & $5.7 \pm 0.2^{\mathrm{a}}$ & $23.3 \pm 0.6^{\mathrm{d}}$ & $34.6 \pm 2.0^{\mathrm{b}}$ & $1912 \pm 18$ \\
Tamazula & $6.1 \pm 0.4^{\mathrm{a}}$ & $33.3 \pm 0.4^{\mathrm{b}}$ & $16.5 \pm 0.5^{\mathrm{d}}$ & $1313 \pm 10$ \\
Zapotlán el Grande & $5.0 \pm 0.3^{\mathrm{bc}}$ & $27.9 \pm 0.3^{\mathrm{c}}$ & $42.9 \pm 1.3^{\mathrm{a}}$ & $1512 \pm 21$ \\
Sayula & $5.5 \pm 0.3^{\mathrm{abc}}$ & $36.5 \pm 0.6^{\mathrm{a}}$ & $36.7 \pm 1.1^{\mathrm{b}}$ & $1425 \pm 38$ \\
San Martín Hidalgo & $4.3 \pm 0.2^{\mathrm{c}}$ & $34.2 \pm 0.7^{\mathrm{a}, \mathrm{b}}$ & $13.8 \pm 0.6^{\mathrm{e}}$ & $1317 \pm 12$ \\
Zacoalco & $5.3 \pm 0.2^{\mathrm{abc}}$ & $31.9 \pm 0.9^{\mathrm{b}}$ & $44.1 \pm 2.6^{\mathrm{a}}$ & $1455 \pm 12$ \\
& & & & \\
\hline
\end{tabular}

Letras diferentes indican diferencias significativas entre municipios en base a análisis de varianza y pruebas de Fisher. 
Tabla 3. Medias \pm E.E. de cantidad de cría (número panales) y de factores climáticos y ambientales (altura sobre el nivel del mar: asnm) de las colonias de abejas evaluadas para comportamiento higiénico por región

\begin{tabular}{lcccc}
\hline Región & Panales c/cría & Temperatura & Humedad Rel. & asnm \\
\hline Llano & $5.2 \pm 0.1^{\mathrm{a}}$ & $33.2 \pm 0.4^{\mathrm{a}}$ & $30.2 \pm 1.5^{\mathrm{a}}$ & $1400 \pm 13$ \\
Montaña & $5.3 \pm 0.2^{\mathrm{a}}$ & $26.2 \pm 0.5^{\mathrm{b}}$ & $29.5 \pm 1.3^{\mathrm{a}}$ & $2016 \pm 20$ \\
\hline
\end{tabular}

Letras diferentes indican diferencias significativas entre regiones en base a pruebas t de student.

Se encontraron correlaciones significativas entre el grado de comportamiento higiénico de las colonias, la temperatura ambiental y la asnm. Sin embargo, no hubo correlación significativa del comportamiento higiénico de las colonias ni con la cantidad de cría ni con la humedad relativa (Tabla 4 ).

Tabla 4. Correlaciones e intervalo de confianza (I. C. 95\%) entre el nivel de comportamiento higiénico (C. H.) de las colonias de abejas evaluadas y factores climáticos, ambientales y panales con cría

\begin{tabular}{lccr}
\hline Características correlacionadas & $\mathbf{r}$ & $\mathbf{p}$ & I. C. 95\% \\
\hline C. H. - Temperatura & & & $0.09-0.40$ \\
C. H. - Humedad relativa & 0.25 & 0.002 & $-0.15-0.19$ \\
C. H. - Altura sobre nivel del mar & 0.02 & 0.826 & $-0.41-0.12$ \\
C. H. - Panales con cría & -0.27 & 0.001 & $-0.14-0.20$ \\
\hline
\end{tabular}

Analizando todas las colonias evaluadas, el $50 \%$ de ellas tuvieron bajos niveles de comportamiento higiénico $(<60 \%)$, mientras que el 25.3 y $11.3 \%$ de ellas, tuvieron un comportamiento higiénico alto $(>80 \%)$ o muy alto (>95\%) respectivamente. El análisis por regiones reveló que, en la región del llano, un mayor porcentaje de colonias mostró alto comportamiento higiénico $(>80 \%)$ en comparación con las colonias de la región de montaña (34.5 y 10.9\%, respectivamente, Figuras 3a y 3b). Además, en la región del llano, $16.1 \%$ de las colonias mostraron un muy alto comportamiento higiénico (> 95\%) contra solo el $3.6 \%$ de las colonias en la región de montaña. Este estudio mostró que las colonias de abejas ubicadas en los municipios de la región del llano fueron significativamente más higiénicas que las colonias ubicadas en los municipios de la región de montaña y que estas diferencias están relacionadas con la temperatura ambiental y la asnm. También se encontró que en los municipios más cálidos y de menor altitud, existe una mayor proporción de colonias de abejas con alto o muy alto comportamiento higiénico que en sitios más fríos y de mayor elevación.

\section{DISCUSIÓN}

Aunque hubo cierto grado de variabilidad entre municipios, el número de panales con cría de las colonias de abejas no tuvo relación con la remoción de cría por lo que aparentemente no influyó en el comportamiento higiénico en las poblaciones de abejas 
estudiadas. Este resultado probablemente se debió a una reducida variación en la cantidad de cría de las colonias estudiadas entre regiones. Es posible que en otra época del año estas condiciones pudieran cambiar y que diferencias de panales con cría en las colonias pudieran resultar en diferencias poblacionales que influirían en su grado de comportamiento higiénico. Independientemente de ese posible escenario hipotético, el cual requeriría evaluaciones frecuentes a lo largo del año para demostrarse, el estudio de Medina-Flores et al. (2014a) concuerda con los resultados de este trabajo. MedinaFlores et al. (2014a) congelaron con nitrógeno líquido panales de cría de colonias de abejas en Zacatecas y evaluaron su comportamiento higiénico, no encontrando correlaciones estadísticas entre remoción de cría muerta y número de panales con cría.

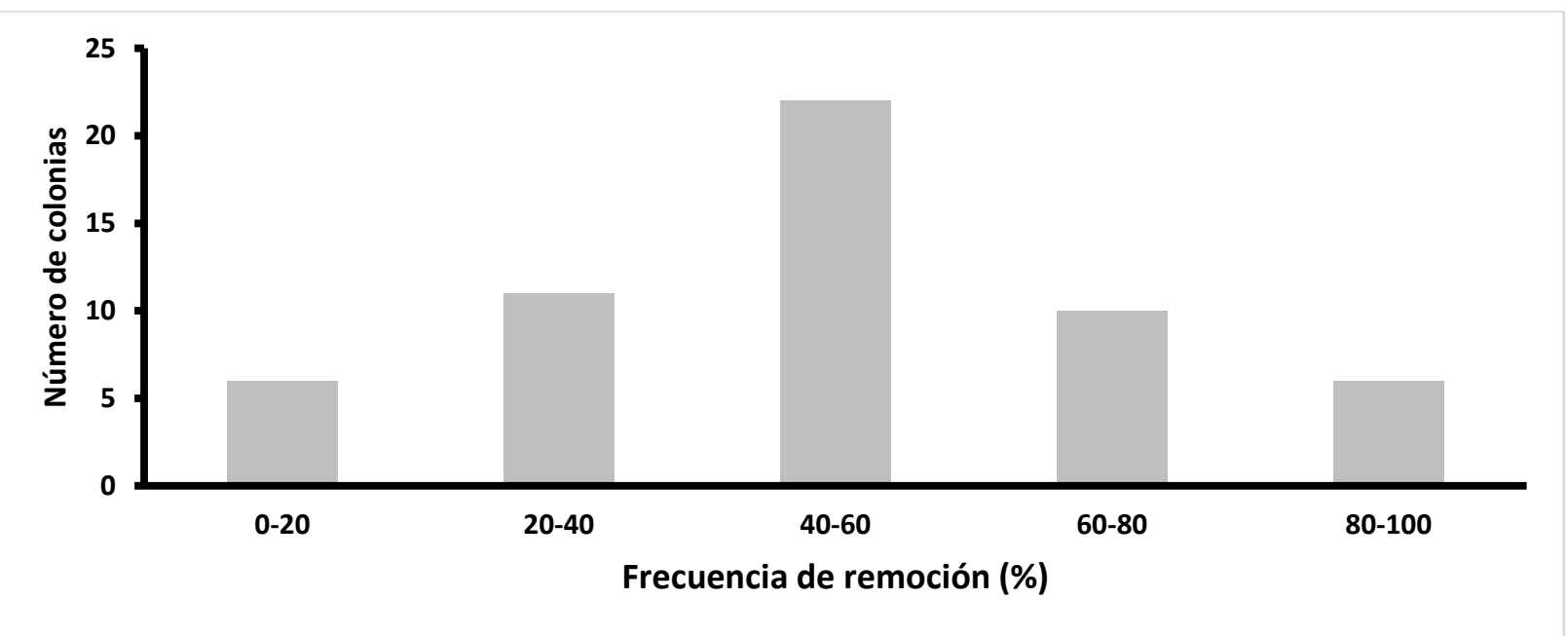

Figura 3a. Frecuencia de colonias con diferentes porcentajes de remoción de cría en la zona de montaña $(n=55)$

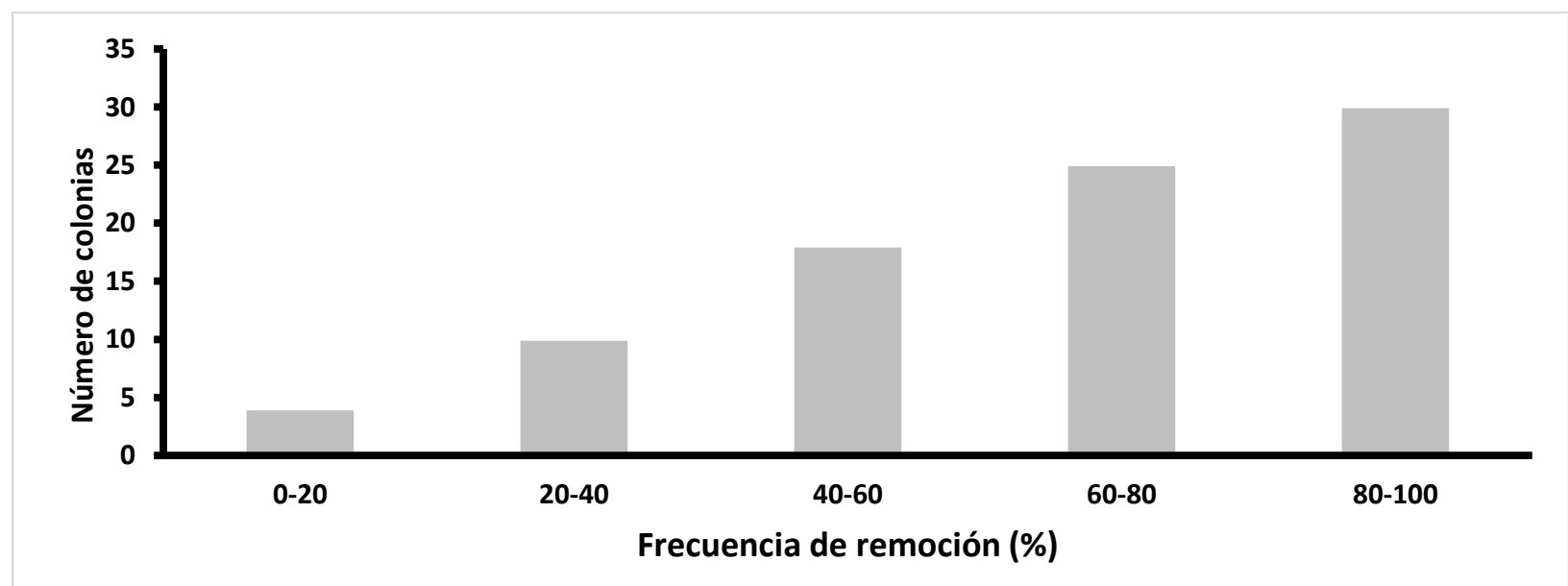

Figura 3b. Frecuencia de colonias con diferentes porcentajes de remoción de cría en la zona del llano $(\mathrm{n}=\mathbf{8 7})$ 
Dentro de los factores climáticos, la humedad relativa no tuvo correlación significativa con el comportamiento higiénico, pero se encontró una correlación positiva y significativa entre temperatura ambiental y remoción de cría muerta, lo que indica que a medida que aumenta la temperatura también aumenta la remoción de cría. En un estudio similar llevado a cabo en Brasil a distintas altitudes y con abejas africanizadas, también se encontró una correlación positiva entre temperatura y remoción de cría (Sousa et al., 2016). Por su parte Cheruiyot et al. (2017) señalan que las variables climáticas podrían ejercer una influencia sobre el comportamiento higiénico. Es posible que la temperatura y la africanización favorezcan una mayor actividad motora de las abejas y eso facilite la remoción de cría muerta, pero esta hipótesis tendría que probarse en futuros estudios, ya que no fue el objetivo de esta investigación.

El comportamiento higiénico de las poblaciones de abejas estudiadas también se correlacionó, aunque negativamente, con la asnm, lo que indica que a medida que los apiarios estaban ubicados a mayor altura (en un rango de asnm de 1235 a $2248 \mathrm{~m}$ ), menor fue el grado de comportamiento higiénico de las colonias. Además, al comparar las regiones, las colonias ubicadas en la región del llano fueron significativamente más higiénicas que las colonias de la región de montaña. Lo anterior es corroborado por un menor porcentaje de colonias con alto comportamiento higiénico en la región de montaña (11\%), mientras que, en la región del llano, el porcentaje de colonias altamente higiénicas fue mayor (>34\%). Estas diferencias pueden ser probablemente atribuibles a una mayor incidencia de abejas con ancestría africana (Apis mellifera scutellata) en la zona del llano, ya que las abejas africanizadas son más higiénicas y poseen mayor capacidad de detectar, desopercular y retirar cría enferma o infestada con $V$. destructor que abejas de razas europeas (Pereira et al., 2013; Medina-Flores et al., 2014b Nganso et al., 2017).

Está demostrado que el grado de africanización de las poblaciones de abejas en México muestra un gradiente contínuo asociado a la asnm. A menores altitudes, el grado de africanización de las colonias es mayor que en zonas de montaña (Medina-Flores et al., 2015; Domíngues-Ayala et al., 2016; Guzmán-Novoa et al., 2020) y las colonias más africanizadas en México manifiestan menores niveles de infestación por $V$. destructor y enfermedades virales y de la cría, en parte debido al comportamiento higiénico de las abejas (Guzman-Novoa et al., 2012, 2013; Medina-Flores et al., 2014a, b, c; Hamiduzzaman et al., 2015), aunque en ocasiones, a pesar de su alto comportamiento higiénico, una combinación de parasitosis como la varroosis y la ascosferosis pueden hacer colapsar colonias de abejas africanizadas (Medina y Vicario-Mejía, 1999). Una evidencia adicional que apoya la presunción de mayor grado de africanización de las colonias en la región del llano, son los resultados del estudio de Esquivel et al. (2015), quienes mostraron que las abejas de colonias de la región del llano eran más africanizadas y defensivas que las abejas de colonias de la región de montaña. 
El hecho de haberse encontrado que más del $25 \%$ de las colonias evaluadas tuvieron > $80 \%$ de comportamiento higiénico y que más del $11 \%$ de ellas mostraron grados de comportamiento higiénico $>95 \%$, sugiere que estos porcentajes de remoción de cría son aceptables para iniciar proyectos de crianza selectiva de abejas altamente higiénicas para mejorar la sanidad de las colonias. Estos porcentajes son mayores que los reportados en otros trabajos como el realizado por Medina-Flores et al. (2014c) en el estado de Zacatecas, donde el promedio de remoción de cría a las 48 h fue de $75 \%$ en las colonias más higiénicas. Masaquiza et al. (2017) estudiando colonias de abejas africanizadas en Ecuador, encontraron porcentajes de remoción de cría del $76 \%$ utilizando el método de pinchado con aguja, método que sobre-estima el comportamiento higiénico de las abejas (Espinosa-Montaño et al., 2008). Por lo anterior, puede concluirse que los resultados encontrados en este trabajo son importantes debido a que la potencial reproducción de genotipos de abejas con alto comportamiento higiénico, contribuye a aumentar la resistencia de las colonias a enfermedades (Guzman-Novoa y Morfin, 2019), lo cual es útil para reducir el uso de antibióticos en la prevención y tratamiento de brotes de enfermedades de la cría; asimismo, las colonias higiénicas tendrían menor cantidad de ácaros de $V$. destructor que aquellas no seleccionadas (Spivak, 1996) y pudieran ser capaces de mantenerse saludables a pesar de la existencia de colonias infestadas en el mismo apiario (Russo et al., 2020). Adicionalmente a la resistencia a enfermedades y ácaros parasitarios, el comportamiento higiénico de las abejas también ha sido relacionado con un efecto positivo en la producción de miel (Wielewski et al., 2012; Medina-Flores et al., 2014c).

Varios estudios han demostrado que el comportamiento higiénico de las abejas está fuertemente influenciado por efectos genéticos y que al menos siete loci lo controlan (Arechavaleta-Velasco et al., 2011). El índice de heredabilidad para esta característica se ha estimado entre 0.17 y 0.63 (Boecking et al., 2000; Pernal et al., 2012), por lo que es viable desarrollar estirpes de abejas higiénicas en programas de mejoramiento genético. De hecho, Spivak (1996) produjo dos líneas de abejas para alto y bajo comportamiento higiénico. Además, el comportamiento higiénico se hereda principalmente por la vía materna (Unger y Guzman-Novoa, 2010), por lo que se esperaría progreso genético en la progenie de futuras generaciones aun cuando las reinas higiénicas sean fecundadas por zánganos de poblaciones no higiénicas (Ivernizzi et al., 2011). Todo lo anterior permite inferir que, si se inician programas de crianza selectiva con colonias en las regiones estudiadas, se produciría mejoramiento genético generación tras generación en beneficio de los apicultores. La selección de colonias en la región de montaña usaría una base de abejas más europeizada, mientras que la selección de colonias en la región del llano lo haría con abejas con mayor grado de africanización. De implementarse estos programas, también habría que considerar que las abejas africanizadas producen menos miel y son más defensivas que las europeas (Uribe-Rubio et al., 2003; Guzmán-Novoa y Uribe-Rubio, 2004). 


\section{CONCLUSIÓN}

El grado de comportamiento higiénico de las poblaciones de abejas estudiadas varía en relación a condiciones climáticas y ambientales. Colonias establecidas en regiones más cálidas y a menor altitud exhibieron mayores grados de comportamiento higiénico en comparación con colonias establecidas en zonas más frías y a mayor altitud. También se encontró que más del $25 \%$ de las colonias evaluadas tuvieron un alto grado de comportamiento higiénico (> $80 \%$ ), lo que permitiría iniciar proyectos de crianza selectiva de abejas altamente higiénicas para mejorar la sanidad de las colonias, lo cual beneficiaría a la industria apícola.

\section{IMPLICACIONES}

Las implicaciones de este estudio al evaluar y caracterizar el comportamiento higiénico de colonias de abejas en diversos municipios del estado de Jalisco de acuerdo a sus condiciones climáticas, favorece iniciar proyectos de crianza selectiva de abejas en beneficio del sector apícola.

\section{AGRADECIMIENTOS}

Se agradece a la Asociación de Apicultores y Polinizadores de Jalisco por su apoyo al facilitar el uso de las colonias y apiarios estudiados en este trabajo.

A todos los colaboradores en la recolección de muestras.

\section{LITERATURA CITADA}

ARECHAVALETA-VELASCO ME, Hunt GJ, Spivak M, Camacho-Rea C. 2011. Loci de rasgos binarios que influyen en la expresión del comportamiento higiénico de las abejas melíferas. Revista Mexicana de Ciencias Pecuarias 2 (3): 238-298. ISSN: 2007-1124. http://www.scielo.org.mx/scielo.php?script=sci_arttext\&pid=S2007-11242011000300004

BOECKING O, Bienefeld K, Drescher W. 2000. Heritability of the Varroa-specific hygienic behavior in honey bees (Hymenoptera: Apidae). Journal of Animal Breeding and Genetics. 117 (6): 417-424. ISSN: 1439-0388. https://doi.org/10.1046/j.14390388.2000.00271.x

CHERUIYOT S, Lattorff H, Kahuthia-Gathu R, Mbugi J, Muli E. 2018. Varroa-specific hygienic behavior of Apis mellifera scutellata in Kenya. Apidologie, 49(4): 439-449. https://doi.org/10.1007/s13592-018-0570-6

CONTRERAS-ESCAREÑO F, Pérez AB, Echazarreta CM, Cavazos AJ, Macías-Macías JO, Tapia-González JM. 2013. Características y situación actual de la apicultura en las regiones Sur y Sureste de Jalisco, México. Revista Mexicana de Ciencias Pecuarias. 4(3): 387-398. ISSN: 2007-1124.

http://www.scielo.org.mx/scielo.php?script=sci_arttext\&pid=S2007-11242013000300009 
DELAPLANE KS, van der Steen J, Guzman-Novoa E. 2013. Standard methods for estimating strength parameters of Apis mellifera colonies. Journal of Apicultural Research. 52: 1-12. ISSN: 0021-8839. https://www.tandfonline.com/doi/pdf/10.3896/IBRA.1.52.1.03

DOMÍNGUEZ-AYALA R, Moo-Valle H, May-Itzá WJ, Medina-Peralta S, Quezada-Euán JJG. 2016. Stock composition of northern Neotropical honey bees: mitotype and morphotype diversity in Mexico (Hymenoptera: Apidae). Apidologie. 47: 642-652. ISSN: 0044-8435. https://doi.org/10.1007/s13592-015-0414-6

EMSEN B, Petukhova T, Guzman-Novoa E. 2012. Factors limiting the growth of Varroa destructor populations in selected honey bee (Apis mellifera) colonies. Journal of Animal and Veterinary Advances. 11: 4519-4525. ISSN:1680-5593.

https://www.researchgate.net/publication/287716219_Factors_Limiting_the_Growth_of_ Varroa_destructor_Populations_in_Selected_Honey_Bee_Apis_mellifera_L_Colonies

ESPINOSA-MONTAÑO LG, Guzman-Novoa E, Sánchez-Albarrán A, Montaldo HH, Correa-Benítez A. 2008. Estudio comparativo de tres pruebas para evaluar el comportamiento higiénico en colonias de abejas (Apis mellifera L.). Veterinaria. México 39: 39-54. ISSN: 0301-5092.

http://www.scielo.org.mx/scielo.php?script=sci_arttext\&pid=S0301-50922008000100004

ESQUIVEL R, Macías-Macías J, Tapia-González J, Contreras-Escareño F, de León Mantecón M, Silva-Contreras A. 2015. Selección de abejas (Apis mellifera L) con baja defensividad y su relación con el ambiente en Jalisco, México. Abanico Veterinario. 5(1): 44-50. ISSN: 2448-6132. http://www.scielo.org. $\mathrm{mx} / \mathrm{scielo} . \mathrm{php}$ ? $\mathrm{pid}=\mathrm{S} 2448-$ 61322015000100044\&script=sci_arttext

GOBIERNO DEL ESTADO DE JALISCO. 2019. Municipios de Jalisco. https://www.jalisco.gob.mx/jalisco/municipios

GUZMAN-NOVOA E, Uribe-Rubio JL. 2004. Honey production by European, Africanized and hybrid honey bee (Apis mellifera) colonies in Mexico. American Bee Journal. 144: 318-320. ISSN: 0002-7626. https://dx.doi.org/10.1016/B978-0-444-64046-8.00254-8

GUZMAN-NOVOA E, Hamiduzzaman MM, Espinosa-Montaño L, Correa-Benítez A, Anguiano-Baez R, Ponce-Vázquez R. 2012. First detection of four viruses in honey bee (Apis mellifera) workers with and without deformed wings and Varroa destructor in Mexico. Journal of Apicultural Research. 51: 342-346. ISSN: 0021-8839.

https://doi.org/10.3896/IBRA.1.51.4.08 
GUZMAN-NOVOA E, Hamiduzzaman MM, Correa-Benítez A, Espinosa-Montaño LG, Uribe-Rubio JL. 2013. A scientific note on the first detection of black queen cell virus in honey bees (Apis mellifera) in Mexico. Apidologie. 4: 382-384. ISSN: 0044-8435. https://doi.org/10.1007/s13592-012-0191-4

GUZMAN-NOVOA E, Morfin N. 2019. Disease resistance in honey bees (Apis mellifera L.) at the colony and individual levels. In: Moo-Young, M. (Ed.), Comprehensive Biotechnology. Vol. 4 (67). Elsevier: Pergamon. Pp. 811-817. ISBN-10: 0444533524. https://doi.org/10.1016/B978-0-444-64046-8.00254-8

GUZMAN-NOVOA E., Morfin N, De la Mora A, Macías-Macías JO, Tapia-González JM, Contreras-Escareño F, Medina-Flores CA, Correa-Benítez A, Quezada-Euán JJG. 2020. The process and outcome of the africanization of honey bees in Mexico: lessons and future directions. Frontiers in Ecology and Evolution. 8: 608091. ISSN: 2296-701X. https://doi.org/10.3389/fevo.2020.608091

HANUSZ Z y Tarasińska J. 2015. Normalization of the Kolmogorov-Smirnov and Shapiro-Wilk tests of normality. Biometrical Letters. 52(2): 85-93. https://doi.org/10.1515/bile-2015-0008

HAMIDUZZAMAN MM, Guzman-Novoa E, Goodwin PH, Reyes-Quintana M, Koleoglu G, Correa-Benítez A, Petukhova T. 2015. Differential responses of Africanized and European honey bees (Apis mellifera) to viral replication following mechanical transmission or Varroa destructor parasitism. Journal of Invertebrate Pathology. 126: 12-20. ISSN: 00222011. https://doi.org/10.1016/j.jip.2014.12.004

IVERNIZZI C, Rivas F, Bettucci L. 2011. Resistance to chalkbrood disease in Apis mellifera L. (Hymenoptera: Apidae) colonies with different hygienic behavior. Neotropical Entomology. 40: 28-34. ISSN: 1519-566X. https://doi.org/10.1590/S1519566X2011000100004

MAGAÑA-MAGAÑA M, Tavera-Cortés M, Salazar-Barrientos L, Sanginés-García J. 2016. Productividad de la apicultura en México y su impacto sobre la rentabilidad. Revista Mexicana Ciencias Agrícolas 7: 1103-1115. ISSN: 2007-0934. https://doi.org/10.29312/remexca.v7i5.235

MASAQUIZA D, Curbelo-Rodrígues LM, Díaz-Monroy BL, Pilataxi R, Andrade-Yucailla V. 2017. Comportamiento higiénico y nivel de infestación con Varroa destructor de Apis mellifera en la zona centro del Ecuador. Revista Ecuatoriana de Investigaciones Agropecuarias. 2(1):25-30. ISSN: 2528-8172. https://doi.org/10.31164/reiagro.v2n1.5 
MEDINA LM, Vicario-Mejía E. 1999. The presence of Varroa jacobsoni mite and Ascosphaera apis fungi in collapsing and normal honey bee (Apis mellifera L.) colonies in Yucatan, Mexico. American Bee Journal. 139(10): 794-796. ISSN: 0002-7626. http://www.scopus.com/inward/record.url?eid=2-s2.0-

0033247087\&partnerID=MN8TOARS

MEDINA-FLORES CA, Guzman-Novoa E, Aréchiga C, Gutiérrez-Bañuelos H, AguileraSoto JI. 2014a. Honey production and Varroa destructor infestation of Africanized honey bee (Apis mellifera) colonies with high and low hygienic behavior. Revista Mexicana de Ciencias Pecuarias 5(2): 157-170. ISSN: 2007-1124.

https://doi.org/10.22319/rmcp.v5i2.3222

MEDINA-FLORES CA, Guzman-Novoa E, Hamiduzzaman MM, Aréchiga-Flores C, López-Carlos M. 2014b. Africanized honey bees (Apis mellifera) have low infestation levels of the mite Varroa destructor in different ecological regions in Mexico. Genetics and Molecular Research. 13: 7282-7293. ISSN: 1676-5680.

https://doi.org/10.4238/2014.February.21.10

MEDINA-FLORES CA, Guzman-Novoa E, Espinosa-Montaño LG, Uribe-Rubio JL, Gutierrez-Luna R, Gutierrez-Piña F. 2014c. Frequency of varroosis and nosemosis in honey bee (Apis mellifera) colonies in the state of Zacatecas, Mexico. Revista Chapingo Serie Ciencias Forestales y del Ambiente. 20: 159-167. ISSNe: 2007-4018. https://doi.org/10.5154/r.rchscfa.2013.08.028

MEDINA-FLORES CA, Guzman-Novoa E, Hamiduzzaman MM, Aguilera-Soto J, LópezCarlos MA. 2015. Africanization of honey bees (Apis mellifera) in three climatic regions of northern Mexico. Veterinaria México. 2(4): 1-9. ISSN: 0301-5092. https://doi.org/10.21753/vmoa.2.4.353

MEDINA-FLORES CA, Guzmán-Novoa E, Aguilera JI, López MA, Medina-Cuéllar SE. 2019. Condiciones poblacionales y alimenticias de colonias de abejas melíferas (Apis mellifera) en tres regiones del altiplano semiárido de México. Rev. mex. de cienc. Pecuarias. 10(1): 199-211. https://doi.org/10.22319/rmcp.v10i1.4387

NGANSO BT, Fombong AT, Yusuf A A, Pirk CWW, Stuhl C, Torto B. 2017. Hygienic and grooming behaviors in African and European honeybees-New damage categories in Varroa destructor. PLOS ONE. 12(6): e0179329. ISSN:1932-6203. https://doi.org/10.1371/journal.pone.0179329 
PEREIRA RA, Morais MM, Francoy TM, Gonçalves LS. 2013. Hygienic behavior of Africanized honey bees Apis mellifera directed towards brood in old and new combs during diurnal and nocturnal periods. Insects. 4(4): 521-532. ISSN: 2075-4450. https://doi.org/10.3390/insects4040521

PERNAL SF, Sewalem A, Melathopoulos AP. 2012. Breeding for hygienic behavior in honeybees (Apis mellifera) using free-mated nucleus colonies. Apidologie. 43: 403-416. ISSN: 0044-8435. https://doi.org/10.1007/s13592-011-0105-x

PLAN REGIONAL DE DESARROLLO. 2012. Región 06 Sur de Jalisco. Comité Técnico de Planeación y Evaluación de Jalisco. Gobierno del Estado de Jalisco. Pp. 211. https://transparenciafiscal.jalisco.gob.mx/sites/default/files/plan_de_desarrollo_region_0 6_sur_vp1.pdf

QUEZADA-LUCIO N. 2017. Estadística con SPSS 24. Macro., ISBN: 978-612-304-5487.

RUSSO RM, Liendo MC, Landi L, Pietronave H, Merke J, Fain H, et al. 2020. Grooming behavior in naturally Varroa-resistant Apis mellifera colonies from North-Central Argentina. Frontiers in Ecology and Evolution. 8: 590281. ISSN: 2296-701X. https://doi.org/10.3389/fevo.2020.590281

SOTO-MUCIÑO L, Elizarraras-Baena R, Soto-Muciño I. 2017. Situación apícola en México y perspectiva de la producción de miel en el Estado de Veracruz. Revista de Estrategias del Desarrollo Empresarial. 3(7): 40-64. ISSN: 2444-4952. http://www.ecorfan.org/spain/researchjournals/Estrategias_del_Desarrollo_Empresarial/ vol3num7/Revista_de_Estrategias_del_Desarrollo_Empresarial_V3_N7_5.pdf

SOUSA ARS, Araújo ED, Gramacho KP, Nunes LA. 2016. Bee's morphometrics and behavior in response to seasonal effects from ecoregions. Genetics and Molecular $\begin{array}{lllll}\text { Research. } & 15 & (2): & \text { gmr.15027597. } & \text { ISSN: }\end{array}$ https://doi.org/10.4238/gmr.15027597

SPIVAK M.1996. Honey bee hygienic behavior and defense against Varroa jacobsoni. Apidologie. 27: 245-260. ISSN: 0044-8435. https://doi.org/10.1051/apido:19960407

TAPIA-GONZÁLEZ J, Alcazar-Oceguera G, Macías-Macías J, Contreras-Escareño F, Tapia-Rivera J, Chavoya-Moreno F, Martínez-González J. 2017. Nosemosis en abejas melíferas y su relación con factores ambientales en Jalisco, México. Revista Mexicana de Ciencias Pecuarias 8(3): 325-330. ISSN: 2007-1124. http://dx.doi.org/10.22319/rmcp.v8i3.4510 
TAPIA-GONZÁLEZ J, Alcazar-Oceguera G, Macías-Macías J, Contreras-Escareño F, Tapia-Rivera J, Petukhova T, Guzmán-Novoa E. 2019. Varroosis en abejas melíferas en diferentes condiciones ambientales y regionales de Jalisco, México. Ecosistemas y Recursos Agropecuarios. 6(17): 243-251. ISSN: 2007-9028. https://doi.org/10.19136/era.a6n17.2018

TAPIA-GONZÁLEZ J, Alcazar-Oceguera G, Macías-Macías J, Contreras-Escareño F, Tapia-Rivera J, Petukhova T, Guzmán-Novoa E. 2020. Ascosferosis en abejas melíferas y su relación con factores ambientales en Jalisco, México. Revista Mexicana de Ciencias Pecuarias 11(2): 468-478. ISSN: 2007-1124. https://doi.org/10.22319/rmcp.v11i2.4926

UNGER P, Guzman-Novoa E. 2010. Maternal effects on the hygienic behavior of Russian x Ontario hybrid honeybees (Apis mellifera L.). Journal of Heredity. 101(1) :91-96. ISSN: 0022-1503. https://doi.org/10.1093/jhered/esp092

URIBE-RUBIO JL, Guzmán-Novoa E, Hunt GJ, Correa-Benítez A, Zozaya RJA. 2003. The effect of africanization and honey production, defensive behavior and size of honeybees (Apis mellifera L.) in the Mexican high plateau. Veterinaria México. 34: 47-59. ISSN: 0301-5092. https://www.medigraphic.com/pdfs/vetmex/vm-2003/vm031e.pdf

WAGONER KM, Spivak M, Rueppell O. 2018. Brood affects hygienic behavior in the honey bee (Hymenoptera: Apidae). Journal of Economic Entomology. 111: 2520-2530. ISSN: 0022-0493. https://doi.org/10.1093/jee/toy266

WIELEWSKI P, de Toledo VAA, Nunes ME, Costa-Maia FM, Faquinello P, Lourenco DAL, Ruvolo-Takasusuki MCC, Oliveira, CAL, Sereia MJ. 2012. Relationship between hygienic behavior and Varroa destructor mites in colonies producing honey or royal jelly. Sociobiology. $59(1)$ 251-274. ISSN: http://dx.doi.org/10.13102/sociobiology.v59i1.682

WILLIAM H, Ahrens, Darrell J, Girish Budhwar. 1990. Use of the Arcsine and Square Root Transformations for Subjectively Determined Percentage Data. Weed Science, 38(4): 452-458. http://www.jstor.org/stable/4044902

XONIS C, Thrasyvoulou A, EI Taj HF. 2015. Variability of hygienic behavior in bee Apis mellifera macedonica. Bulgarian Journal of Agricultural Science. 21(3): 680-685. ISSN: 1310-0351. https://www.agrojournal.org/21/03-30.pdf 